\title{
Research on Smart Regulation of Intelligent Connected Vehicles
}

\author{
Yongqin Feng ${ }^{1}$, Xiaorui Zhang ${ }^{1, *}$, Wenzhao $\mathrm{Li}^{1}$, Le Liu ${ }^{1}$ \\ ${ }^{1}$ Sub-Institute of Product Safety, China National Institute of Standardization, Beijing, 100800, China \\ "Corresponding author: Email: zhangxr@dpac.gov.cn
}

\begin{abstract}
Safety is the key to the sustainable and healthy development of intelligent connected vehicle industry. This study analyzed the characteristic of smart regulation of intelligent connected vehicles abroad. In view of the development and safety supervision of intelligent connected vehicles in China, this study put forward the suggestions of smart regulation of intelligent connected vehicles, including improving and perfecting data security laws and regulations, establishing information platform of safety supervision, and strengthening the technical support systems for safety supervision.
\end{abstract}

Keywords: Smart regulation, Intelligent connected vehicles, Safety.

\section{INTRODUCTION}

At present, the new generation of information technology represented by big data, cloud computing and artificial intelligence deeply integrated with the real economy. The market of intelligent connected vehicles is growing rapidly. According to the 'Industry Impact Outlook of the 14th Five-Year Plan-Manufacturing and Automobiles' released by KPMG, it is estimated that by 2025, the sales volume of PA(partially autonomous driving) and $\mathrm{CA}$ (Conditional autonomous driving) intelligent connected vehicles in China will account for more than $50 \%$ of the total sales volume. While the technology of intelligent connected vehicles is constantly changing, new technical defects and safety risks, such as fire, information and network security, also caused, posing severe challenges to industrial transformation and upgrading and government supervision. Against this background, it has become a key problem and practical need to explore the product safety supervision matching intelligent connected vehicles.

Smart Regulation is a new regulation theory developed in the 1990s. It was first proposed by Gunningham and Grabosky in Smart regulation: Designing environmental policy. Smart Regulation refers to a diversified form of regulation that incorporates flexible, imaginative and innovative forms of social control [1]. Subsequently, they put forward eight principles of smart regulation including: avoid "perverse" or adverse effects of adjoining policies; choose policy mixes that incorporate a broad range of instruments, etc. [2]. Smart regulation theory has been widely used in environmental governance, financial supervision, food supervision and other fields. It has gradually become a new trend of global regulatory reform. Jordan et al. proposed policy portfolio tools such as optimizing government-funding plans and strengthening the coordination between innovation promoters and innovation laboratories based on the theory of smart regulation [3]. Liu et al. proposed that the framework of smart regulation theory includes supervision concept, supervision subject, supervision means and supervision effect analysis [4]. Zhang et al. introduced the smart regulation theory to construct a new market regulation mode, and analyzed the regulatory bodies, combined application of policy tools, and sharing of regulatory information based on the "Internet plus" information platform. These studies provide theoretical basis and analytical framework for exploring and establishing the smart regulation of intelligent connected vehicles in China [5].

This study will apply the smart regulation theory; draw lessons from the supervision experience of intelligent connected vehicles abroad, and put forward suggestions on the supervision of intelligent connected vehicle in China, so as to provide support for the healthy and sustainable development of intelligent connected vehicle industry. The structure of this paper is as follows. In the second part, we analyzed the 
characteristics of smart regulation by investigating supervision mode of intelligent connected vehicles abroad. In the third part, we analyzed the development and safety supervision of intelligent connected vehicles in China, and put forward the regulatory challenges faced by intelligent connected vehicles in China. Then, we put forward some suggestions on the smart regulation of intelligent connected vehicles in China. Finally, we summarized the main study conclusions of this paper.

\section{SMART REGULATION OF INTELLIGENT CONNECTED VEHICLES ABROAD}

In order to cope with the challenge of product security to the intelligent connected vehicles, the United States, the European Union and other countries and regions have made a forward-looking strategic layout in frontier technology research and development and product safety. Some of them have explored and established a smart regulation with the supervision information platform as the link and the coordination between government departments and relevant supervision bodies.

\subsection{Incorporate smart connected vehicle security into national security strategy}

Countries around the world regard economic digitization as an important driving force to achieve innovative development, the security of intelligent connected vehicles has risen to the level of national security. The US Congress proposed the Future of Artificial Intelligence Act in 2017, requiring manufacturers to develop detailed network security plans and follow the network security guidance of the National Highway Traffic Safety Administration (NHTSA). In 2015, the European Commission issued the Digital Single Market Strategy to accelerate the development of data protection rules in Europe. The General Data Protection Regulation published in 2016, establishing the LEGAL framework for privacy and data protection in the EU.

\subsection{Take the intelligent connected vehicle as the commanding point of science and technology strategy}

As an important frontier innovation driven by a new round of science and technology, intelligent connected vehicles have become the commanding heights of scientific and technological competition. As the NHTSA released in 2020, Ensuring American Leadership in Automated Vehicle Technology: Automated Vehicles 4.0 clearly seeks to ensure a consistent the United States Government(USG) approach to AV technologies, and to detail the authorities, research, and investments being made across USG so that the United States can continue to lead AV technologies' research, development, and integration[6].

\subsection{Improve the legal system for the safety supervision of intelligent connected vehicle}

Due to the technical characteristics of intelligent connected vehicle, when the intelligent connected vehicle damaged due to the data supply error or failure to supply data, it faces the determination of product liability. This has led states to reassess existing principles of safety and distribution of liability, as well as the applicable conditions for compensation for related damages. For example, Germany has amended the Street Traffic Law to require that autonomous driving vehicles must controlled by human drivers at any time. The UK government is proposing to amend insurance laws for the possible deployment of autonomous vehicles.

\subsection{Establish information platform of safety supervision for intelligent connected vehicle}

In view of the characteristics of digitalization and interconnection of intelligent connected vehicle, information platform for safety supervision of intelligent connected vehicle has established. NHTSA has established a Vehicle Cyber security Response Process for Incidents Involving Safety-Critical Systems. During a significant incident, coordination will handled through DHS's National Cyber security \& Communications Integration Center (NCCIC), with NHTSA having an information/advisory role and performing its statutory responsibility under the Safety Act [6]. In addition, the United States has established the Automotive Information Sharing and Analysis Center (Auto-ISAC) as an industry-led platform for sharing cyber security information.

\subsection{Develop assessment technology of information security for intelligent connected vehicles}

In view of the emerging technology problems brought by intelligent connected vehicles, the research and development of information security assessment technology is strengthen. The US Environmental Protection Agency (EPA) has begun to formulate regulatory policies on fuel economy, energy consumption and exhaust emissions of autonomous vehicles. NHTSA will conduct research on alternative indicators and safety assessment models for autonomous driving. The European Telecommunication Standards Institute (ETSI) has developed a series of information security standards for intelligent connected vehicles and intelligent transportation systems, including ITS security service architecture, communication security 
architecture and security management, trust and privacy management, access control and security services, etc.

\section{REGULATORY OF INTELLIGENT CONNECTED VEHICLES IN CHINA}

\subsection{Overview of the development of Intelligent connected vehicles}

As a key field of China's national intelligent manufacturing development, a series of policy has been issued to guide the development of domestic intelligent vehicle industry. In February 2020, the National Development and Reform Commission and 11 other ministries and commissions jointly issued the Intelligent Vehicle Innovation and Development Strategy. In December 2020, the Ministry of Transport issued the Guidance on Promoting the Development and Application of Road Traffic Autonomous Driving Technology. In November 2020, The National Innovation Center for Intelligent and Connected Vehicles announced the "Intelligent and Connected Vehicle Technology Roadmap 2.0", which greatly promoted the development of intelligent connected vehicles in China.

According to the Report released by China Industry Innovation Alliance for the Intelligent and Connected Vehicles (CAICV), in 2020, the sales volume of L2 class intelligent connected vehicles equipped with automatic emergency braking or adaptive cruise function and lane-keeping system was 3.03 million, with a year-on-year growth of $107 \%$. In terms of power type distribution, the sales volume of new energy intelligent connected vehicles was 316,000 , accounting for $10.4 \%$; the sales volume of oil-fuelled intelligent connected vehicles was 2.716 million, accounting for $89.6 \%$ of the total [7].

From January to December in 2020, the overall sales volume of new energy intelligent connected passenger vehicles showed a sharp upward trend. The proportion of new energy intelligent connected passenger vehicles sales also showed an overall upward trend, accounting for the highest proportion in November, reaching 15.1\%.

\subsection{Safety regulation of intelligent connected vehicles}

In order to promote the safe and healthy development of intelligent connected vehicles, China has been exploring new ways of product safety supervision. In 2019, the State Administration for Market Regulation issued the Notice on Further Strengthening the Management of New Energy Vehicle Recall and the Supplementary Notice on Further Standardizing the Accident Reporting of New Energy Vehicles, establishing the accident reporting and investigation mechanism of new energy vehicles. In
December 2020, the State Administration for Market Regulation issued the Notice on Strengthening Supervision of Over-the-Air (OTA) Technology Recall to regulate the application of OTA technology in recall.

According to the Circular of The State Administration for Market Regulation on The Recall of Vehicles and Consumer Products in China in 2020, the Defective Product Administrative Center of the State Administration for Market Regulation received 37,377 vehicle defect clues from consumers, among which 1,173 defect clues of new energy vehicle reported. The problems of power battery, motor and electronic control system accounted for $49.4 \%$ of the defects of new energy vehicles. In 2020, the number of new energy vehicles recalled was 45 in China, involving 357,000 vehicles, accounting for $5.3 \%$ of the total volume of vehicles recalled in the whole year. Among them, 112,000 vehicles recalled due to defects in the EIC system, accounting for $31.3 \%$ of the total number of new energy vehicles recalled [8].

\subsection{Challenges in the safety regulation of intelligent connected vehicles}

As vehicles become more and more intelligent and networked, in addition to security problems caused by hardware and software defects, information security problems, such as software security risks caused by vehicle intelligence, become increasingly prominent. With the improvement of the degree of intelligence, the amount of software code data in vehicles shows a geometric increase, and the number of code per vehicle reaches more than 100 million lines. For intelligent vehicles calculated by 100 million lines, the software defects should not be underestimated.

At present, facing the technical defects and security risks brought by the rapid development of intelligent connected vehicles, China has not established a perfect governance framework and effective supervision means. In defect investigation, it is urgent to improve the supervision means and analysis ability to confirm the cause of defects quickly.

Data production, collection, processing and analysis are very important for the operation of intelligent connected vehicles. The accident investigation of intelligent connected vehicles increasingly relies on remote data of the vehicle involved. Automobile manufacturers have natural advantages in analyzing faults and accidents based on data, and the "data barrier" to regulatory authorities is increasingly obvious. Even when regulators have access to vehicle data, they lack the tools and expertise to analyze it. At the same time, in the face of numerous and miscellaneous data, it is impossible to effectively supervise and evaluate the authenticity and sufficiency of the materials submitted by manufacturers. 


\section{SUGGESTIONS ON SMART REGULATION OF INTELLIGENT CONNECTED VEHICLES IN CHINA}

Under the background of the rapid development of intelligent connected vehicles, we should make a forward-looking strategic layout for the development of intelligent connected vehicles. Exploring the smart regulation of intelligent connected vehicles is not only an important measure for deepening the reform to streamline the government, delegate power, and improve government services, but also an important path to promote the modernization of the national governance system and governance capability.

\subsection{Improve and perfect the laws and regulations}

From the strategic perspective of ensuring national data security, it is that a safety regulatory system covering the whole life cycle of intelligent connected vehicles data should establish. In view of the technical characteristics of intelligent connected vehicles, establish and improve the laws and regulations of safety supervision of intelligent connected vehicles, and build a security protection wall of terminal data of intelligent connected vehicles.

\subsection{Establish information platform of safety supervision}

Information platform for safety supervision of intelligent connected vehicles should be constructed, using the new generation of information technology represented by artificial intelligence, cloud computing and Internet. This will help realize information sharing between departments, such as transportation, ecological environment, business departments cooperate with each other and give full play to their respective advantages of safety supervision work mechanism and regulatory for intelligent connected vehicles together.

\subsection{Strengthen the technical support systems for safety supervision}

The technical support systems for product safety and recall system of intelligent connected vehicles should strengthen. Accelerate the formation of a technical support system that can quickly respond to the safety supervision of intelligent connected vehicles by strengthening the construction of technical support capacity such as information collection and defect analysis, technical investigation and defect identification, and risk assessment of intelligent connected vehicles. By promoting the construction of national product safety laboratories, focus on solving new safety risks brought by intelligent connected vehicles, research, develop and verify the safety performance indicators of intelligent connected vehicles, develop testing procedures and equipment, and improve the technical capabilities of safety assessment and supervision of intelligent connected vehicle.

\section{CONCLUSIONS}

With the in-depth development of industry technology of intelligent connected vehicle, new technical defects and security risks pose severe challenges to industrial transformation and upgrading and government supervision. Through the review of supervision of intelligent connected vehicle abroad, this study found that some countries have made a forwardlooking strategic layout in frontier technology research and development and product safety, and established information platform for intelligent connected vehicles. Based on the above experience, facing the development of intelligent connected vehicles in China, suggestions on the smart regulation of intelligent connected vehicles are proposed, including the establishment and improvement of safety supervision laws and regulations system covering the whole life cycle of intelligent connected vehicle data. Through the establishment of information platform for safety supervision of intelligent connected vehicle to achieve multidepartment collaborative supervision, and strengthen the technical support systems for safety supervision of intelligent connected vehicles, to improve technology capacity of safety assessment and supervision.

\section{ACKNOWLEDGMENTS}

This research was funded by a dean fund project of China National Institute of Standardization (282020Y7511), and a technology planning project of the State Administration for Market Regulation (2017QK098).

\section{REFERENCES}

[1] N. Gunningham, P. Grabosky, Smart regulation: Designing environmental policy. New York: Oxford University Press. 1998.

[2] P.V.Gossum, A.K.Verheyen, From "smart regulation" to "regulatory arrangements". Policy Sciences, 2010, pp. 245-261. DOI: 10.1007/s110 77-010-9108-0

[3] N.D.Jordan, T.Lemken, C.Liedtke, Barriers to resource efficiency innovations and opportunities for smart regulations the case of Germany. Environmental Policy and Governance, 2014, pp. 307-323. DOI: $10.1002 /$ eet.1632 
[4] P.Liu, L.Wang, The Analysis on the Theory of Responsive Regulation and Its Application in China's Context. Journal of the Renmin University of China, 2016, pp. 91-101.

[5] Y. Zhang, Y.H.Wang, Q.F. Wang, Smart Regulatory Model Based on "Internet Plus" Platform. The Journal of Shanghai Administration Institute, 2020, pp. 18-27.

[6] National Science \& Technology Council, The United States Department Of Transportation. Ensuring American Leadership in Automated Vehicle Technologies: Automated Vehicles 4.0. https://www.transportation.gov/sites/dot.gov/files/2
020-02/EnsuringAmericanLeadershipAVTech4.pdf. 2020.

[7] China Industry Innovation Alliance for the Intelligent and Connected Vehicles. Sales report of China intelligent Internet connected passenger vehicles in 2020. http://www.caicv.org.cn/index. $\mathrm{php} /$ newsInfo?id=386. 2020

[8] Bureau of Quality Development of the State Administration for Market Regulation. Circular of The State Administration for Market Regulation on The Recall of Vehicles and Consumer Products in China in 2020. http://www.samr.gov.cn/zw/zh/ 202103/t20210313_326862.html. 2021 